\title{
Gain Switched Multi-Carrier Transmitter and Pilot Tone Based Receiver for Long Reach Access Networks
}

\author{
Sylwester Latkowski, ${ }^{1}$ Selwan K. Ibrahim, ${ }^{2}$ Kai Shi, ${ }^{1}$ Rui Zhou, ${ }^{1}$ Andrew D. Ellis, ${ }^{1,2}$ \\ Robert Maher, ${ }^{3}$ Liam P. Barry, ${ }^{1}$ and Prince M. Anandarajah ${ }^{1}$ \\ ${ }^{1}$ The Rince Institute, School of Electronic Engineering, Dublin City University, Glasnevin, Dublin 9, Ireland \\ ${ }^{2}$ Photonic Systems Group, Department of Physics and Tyndall National Institute, University College Cork, Ireland \\ ${ }^{3}$ now with Optical Networks Group, University College London, Torrington Place, London WC1E 7JE, UK \\ Author e-mail address: prince.anandarajah@dcu.ie
}

\begin{abstract}
A novel and cost effective long reach PON downlink scenario is proposed employing a multi-carrier transmitter and pilot tone aided direct detection at the receiver. Error free performance with QPSK and $50 \mathrm{~km}$ transmission is presented.

OCIS codes: (060.0060) Fiber optics and optical communications; (060.1660) Coherent communications
\end{abstract}

\section{Introduction}

The explosive growth of the internet, fuelled by the ever-increasing information processing and data creation capability placed at the consumer's disposal, has triggered the introduction of fibre based broadband access networks such as Fibre To The x (FTTx) technology [1] to replace copper based last mile networks. To date, these FTTx networks, have been based on Time Division Multiplexing (TDM) and use dynamic bandwidth allocation algorithms to distribute available capacity amongst users [2]. However, traffic patterns that were once heavily asymmetrical in favour of downloads from network to user are now exhibiting more balance between upstream and downstream bandwidth, as user uploads and peer-to-peer transfers increase. In light of these changes and the requirements imposed by the evolving networks (aggregated bandwidth \& allowable power budget), TDM based access networks may struggle to cope [3]. Hence, Next Generation Access Networks (NGANs) may employ Wavelength Division Multiplexed Passive Optical Network (WDM-PON) technologies that are capable of offering features such as enormous bandwidth, large split ratio, extended transmission reach and enhanced security. Additionally, WDM-PON technology can be combined with advanced modulation formats to increase the information spectral density, thereby improving the overall efficiency of the network. However, there are significant obstacles to the widespread deployment of WDM PONs, such as the high cost and energy consumption of current Optical Line Terminal (OLT) transmitters and Optical Network Unit (ONU) receivers.

In this work, we present a multi-carrier transmitter based on a gain switched discrete mode laser [4] and receiver architecture based on a pilot tone aided direct detection scheme. This simple, robust and cost efficient direct modulation based comb generation technique offers closely spaced coherent channels. The simplified low cost receiver, enabled by the phase correlated optical comb tones, eases the requirement for an expensive local oscillator (tunable laser), negates the need for digital signal processing and also greatly enhances the phase noise tolerance of the system. Experimental work carried out to verify the technique is presented below.

\section{Experimental setup}

The experimental set-up is schematically presented in Fig. 1. Gain switching of the Discrete Mode (DM) laser with an amplified $10.663 \mathrm{GHz}$ sinusoidal signal in conjunction with a dc bias current $(54.7 \mathrm{~mA})$ resulted in a series of seven clearly resolved phase correlated sidebands (3dB of the spectral envelope peak) offset by an integer multiple of the drive frequency. Power equalization and outer sideband rejection was achieved by passing the comb signal through an optical filter (OBPF 1) with both tunable bandwidth and wavelength. This resulted in the unwanted outer sidebands being suppressed to about 15dB below the 6 chosen subcarriers and also yielded comb flatness within a $1 \mathrm{~dB}$ margin as shown in Fig. 2(a). This signal is subsequently optically amplified using an Erbium Doped Fibre Amplifier (EDFA 1) to overcome the loss of the optical filter.

In a practical system, each OLT line card would consist of an optical comb source, an Arrayed Waveguide Grating (AWG) to filter out the required sidebands, an array of modulators to independently encode the data on to each of the sidebands and another AWG to combine them together [5]. However, in this experiment the comb was passed through a dis-interleaver based on Asymmetric Mach Zehnder Interferometers (AMZI), with a free spectral range (FSR) of $21.33 \mathrm{GHz}$, to separate it into even (-2, 0 and 2) and odd (-1, 1 and 3) channels. Both, the odd and even channels were passed through a second stage dis-interleaver to improve the extinction ratio to about 40dB. The two sets of channels were then passed through polarization controllers and Optical Delay Lines (ODLs). The ODLs were used to match the path length of the 2 arms of the interferometer. The 3 odd subcarriers $(-1,1$ and 3$)$ were 
modulated with 2.5Gb/s NRZ electrical data and delayed inverse data patterns, with a pseudo random bit sequence (PRBS) length of $2^{7}-1$, using a Dual Parallel Mach Zehnder Modulator (DPMZM). The data and inverse data streams were de-correlated (11 bit delay) and bit-aligned with the aid of different electrical cable lengths and RF phase shifters. These two de-correlated data signals were then used to drive the in-phase (I) and quadrature (Q) inputs of the DPMZM to achieve a 2.5Gbaud optical Quadrature Phase Shift Keyed (QPSK) signal. The second output of the dis-interleaver (3 even subcarriers: -2, 0 and 2) was un-modulated and passively multiplexed with the 3 data channels with the aid of a polarization maintaining coupler. A $10 \%$ optical tap is passed into a standard Optical Spectrum Analyzer (OSA) with a resolution of $0.02 \mathrm{~nm}$, to enable monitoring of the signals at the output of the OLT.

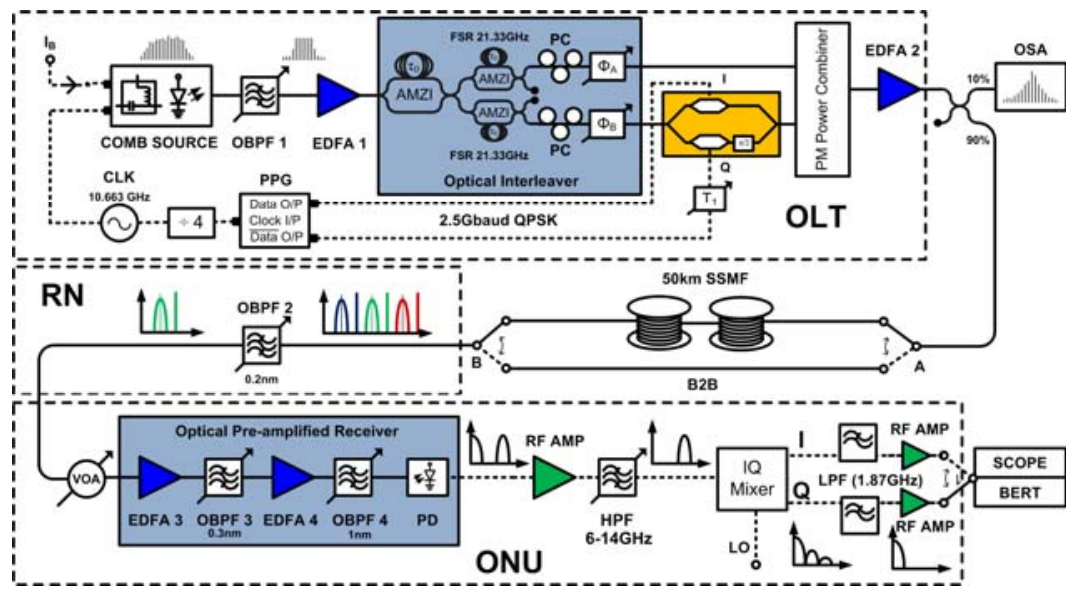

Fig. 1. Experimental configuration of the long reach PON employing a comb source at the OLT and pilot tone based direct detection aided ONU

The multiplexed signal consisting of three 2.5Gbaud QPSK channels and three pilot tones, as shown in Fig. 2 (b), is then transmitted first directly (back-to-back) and subsequently through the feeder fibre to the Remote Node (RN). An optical filter, at the RN, with a $3 \mathrm{~dB}$ bandwidth of $\sim 25 \mathrm{GHz}$ was used to pick out one of the data channels together with an adjacent pilot tone. The output from the RN with the filter tuned to pick out the three different pairs (one pair at a time) is presented in Fig. 2(c). In a practical system the tunable filter would be replaced by an AWG, where each output port would route a pair of signals (channel and pilot tone) to the designated ONU (user premises).
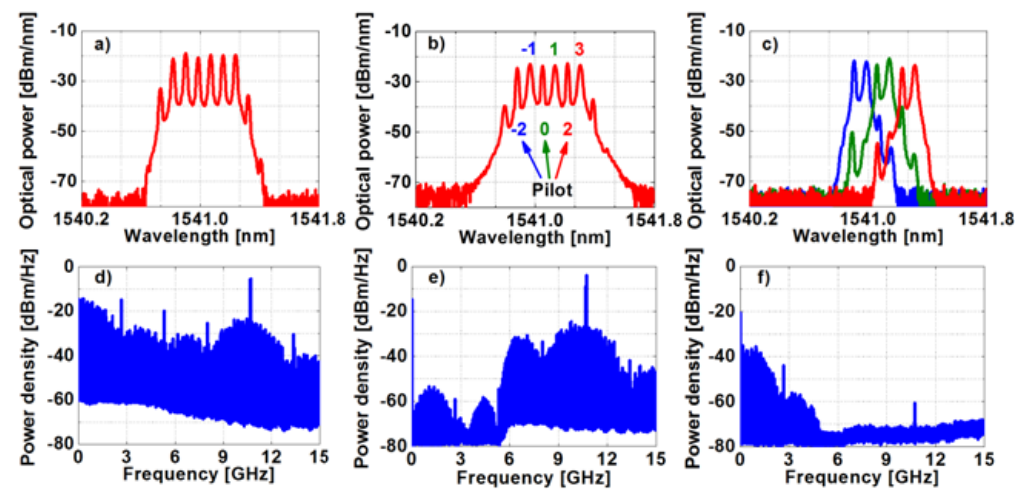

Fig. 2. Optical spectra recorded at $10 \%$ tap of: a) filtered output of the comb source; b) output of the OLT; c) channels filtered out individually with corresponding pilot tone at RN. Electrical spectra acquired in the ONU at: d) the output of the photodiode; e) filtered output after the HPF; f) filtered output of low pass filter after down conversion (LPF).

At the receiver side, a Variable Optical Attenuator (VOA) was used to vary the input power falling on an optically pre-amplified receiver (lack of availability of an appropriate avalanche photodetector) to measure Bit Error Rate (BER) as a function of received power. The receiver consisted of an EDFA (3) acting as a pre-amplifier, a 0.3nm tunable optical bandpass filter (OBPF 3), a second booster EDFA (4) in automatic power control mode, a $1 \mathrm{~nm}$ OBPF (4) and a $40 \mathrm{GHz}$ photodetector. The detected signal was amplified before being high pass filtered (6.3 $\mathrm{GHz}$ cut-off frequency) to reject the detected baseband data and harmonics. This was followed by an IQ mixer for demodulation and a low-pass filter $(1.87 \mathrm{GHz}$ cut-off frequency) to reject the remaining RF signal and LO. A broadband data amplifier was used to boost the signal prior to the error detector and oscilloscope. Electrical spectra 
at various parts of the receiver (at the output of the photodiode, filtered output after the high pass filter, and IQ downconverted and filtered output of low pass filter) are shown in Fig. 2 (d-f).

\section{Experimental Results and Discussion}

The performance of the long reach PON system (downlink) was verified by carrying out BER measurements for two different scenarios: back-to-back (B2B) and 50km Standard Single Mode Fibre (SSMF) transmission. The resulting BER (average of I \& Q) versus received power recorded for each of the three optical data channels is shown in Fig. 3. The red, green and blue lines denote the performance of the centre, right and left channels, respectively, in a B2B scenario. As can be seen in the figure, error-free operation for the outer channels is achieved at $-33 \mathrm{dBm}$ (at BER of $1 \mathrm{e}^{-9}$ ) and for the middle channel at $-32 \mathrm{dBm}$ (at BER of $1 \mathrm{e}^{-9}$ ). The $1 \mathrm{~dB}$ penalty in the case of the middle channel can be mainly attributed to the optical filtering at the RN (leakage of adjacent channels) and to the power asymmetry of the optical comb source. For the sake of clarity, the performance of only the middle channel when transmitted over 50km of SSMF is shown in Fig. 3 and is denoted by the black line. The fibre transmission of the central channel results in a further $1 \mathrm{~dB}$ penalty (relative to the $\mathrm{B} 2 \mathrm{~B}$ performance of the central channel at $1 \mathrm{e}^{-9}$ ) yielding error-free operation at $-31 \mathrm{dBm}$ of received power. The outer channels (left and right) both portrayed a receiver sensitivity (at a BER of $1 \mathrm{e}^{-9}$ ) of $-32 \mathrm{dBm}$. Examples of received eye-diagrams are shown as insets in Fig. 4 , which correspond to both I and Q data recovered from the middle channel for B2B (a and b) and SSMF transmission (c and d) scenarios.

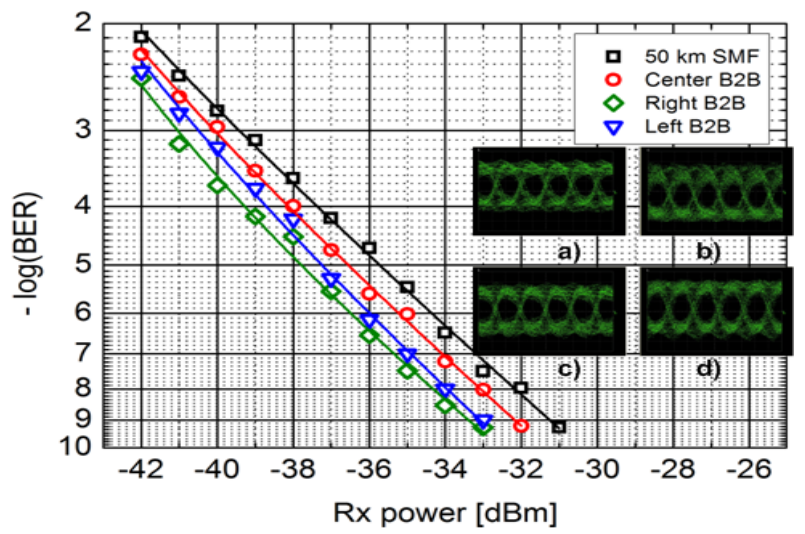

Fig. 3. BER vs received power for 3 individual channels for the B2B scenario and for the center channel after transmission through $50 \mathrm{~km}$ of SSMF. Insets show received eye diagrams of I and Q signals recorded for the center channel in B2B (a \& b) and 50km transmission (c \& d).

\section{Conclusions}

We have demonstrated a low cost multi-carrier transmitter and a novel simplified receiver architecture that could be employed in a long reach PON. The phase correlated, low linewidth optical comb at the OLT transmitter enables dense wavelength channel spacing and facilitates the use of advanced modulation formats. Moreover, the pilot tone aided direct detection scheme at the ONU mitigates the need of a low linewidth optical local oscillator, optical $90^{\circ}$ hybrid and optical phase locking in the customer premises. Experimental work has verified the technique to be capable of transmitting, 2.5Gbaud QPSK data to each user, over 50km of SSMF. This technique, in comparison to coherent detection, compromises on receiver sensitivity, but in return offers simplicity and low cost. Moreover, it can easily be upgraded to accommodate higher order modulation formats, and thus achieve higher data rates.

Acknowledgments: The authors would like to thank Eblana Photonics for the high-speed discrete mode laser. This work was supported in part by the Enterprise Ireland Technology Development Phase Grant 08/324, Science Foundation Ireland Principal Investigator grants 09/IN.1/II2653 and 06/IN/I969, and the Higher Education Authority PRTLI 4 INSPIRE Programs.

\section{References}

[1] P. W. Shumate, “Fibre to the Home: 1977-2007,” IEEE JLT, 26, 1093-1103 (2008)

[2] M. Hajduczenia et. Al.,, “Next Generation PON Systems - Current Status” in Proceedings of ICTON 2009, (IEEE, 2009) Tu.B5.2.

[3] K. Grobre and J-P Elbers, “PON in Adolescence: From TDMA to WDM-PON,” in Communications. Magazine (IEEE, 2008), pp. 26-34

[4] P. M. Anandarajah et al., "Generation of Coherent Multicarrier Signals by Gain Switching of Discrete Mode Lasers,” IEEE Photonics Journal, 3, 112-122 (2011).

[5] S. K. Ibrahim, A.D. Ellis, F.C.G. Gunning, J. Zhao, P. Frascella and F. Peters, "Practical implementation of coherent WDM", in Proc. IEEE Photonics Soc. (LEOS Annual Meeting, 2009), ThM1. 